\title{
THE NUMBER OF FIELD TOPOLOGIES ON COUNTABLE FIELDS
}

\author{
KLAUS-PETER PODEWSKI
}

\begin{abstract}
J. O. Kiltinen proves that every infinite field admits a nondiscrete, Hausdorff field topology. In this note it is shown that every countable field $K$ admits $2^{2{ }^{N_{0}}}$ many field topologies, which even fail to be the join of locally bounded ring topologies.
\end{abstract}

1. Introduction. In $\S 2$ we give a method for generating a fundamental system $\left\{V_{n} \mid n \in \omega\right\}$ of neighborhoods of zero for a field topology on $K$. To do this, we first define the notion of a condition. This is a function from $\omega \times\{0,1\}$ into the set of finite subsets of $K$ with some further properties. A condition $p$ decides for a finite number of elements of $K$ if they are elements of $V_{n}$ or not by saying $r$ is an element of $V_{n}$ if $r \in p(n, 0)$ and $r$ is not an element of $V_{n}$ if $r \in p(n, 1)$. Given two conditions $p$ and $p^{\prime}$, then $p^{\prime}$ extends $p$ if $p(n, i) \subset p^{\prime}(n, i)$ for every $(n, i)$. If $G$ is a chain of conditions, then by the above decision process we get a fundamental system of a field topology.

Since a condition decides only for a finite number of elements of $K$ if they are elements of $V_{n}$ or not, we can prove in $\S 3$ that there are "many" possibilities to extend a condition. In $\S 4$ we define what it means for a set of chains of conditions to be entwined. If $\mathfrak{F}_{1}$ and $\mathfrak{F}_{2}$ are different nonempty subsets of an entwined set then the join-topologies $\bigvee\left\{\mathscr{T}_{G} \mid G \in \mathfrak{G}_{1}\right\}$ and $\bigvee\left\{\mathscr{T}_{G} \mid G \in \mathfrak{G}_{2}\right\}$ are also different. Using the results of $\S 3$ we can construct an entwined set of power $2^{\mathrm{N}}$ of chains of conditions in such a way that for every subset $\mathfrak{F}_{1}$ the topology $\bigvee\left\{\mathscr{T}_{G} \mid G \in \mathfrak{F}_{1}\right\}$ is not the join of locally bounded ring topologies.

2. Chains of conditions. Let $(K,+, \cdot, 0,1)$ be a countable field and let $\phi_{K}$ denote the set of functions $\varphi_{d}, \varphi_{c}, \varphi_{b}$ and $\varphi_{a}, a \in K$, defined by $\varphi_{d}(X)=$ $X /(1-X), \varphi_{c}(X)=X \cdot X, \varphi_{b}(X)=X-X$ and $\varphi_{a}(X)=a \cdot X$, for every subset $X$ of $K$ with $1 \notin X$. Since $K$ is countable there is a sequence $\left(\varphi_{n}\right)_{n \in \omega}$ of elements of $\phi_{K}$ such that the set $\left\{n \mid \varphi=\varphi_{n}\right\}$ is infinite for every $\varphi \in \phi_{K}$. A sequence $\left\{V_{n} \mid n \in \omega\right\}$ of subsets of $K$ is called a fundamental system, if $1 \notin V_{n}, V_{n+1} \subset V_{n}, \varphi_{n}\left(V_{n+1}\right) \subset V_{n}$. For every field topology there is a basic

Received by the editors March 2, 1972 and, in revised form, July 26, 1972. AMS (MOS) subject classifications (1970). Primary $12 \mathrm{~J} 99$.

(c) American Mathematical Society 1973 
system of neighborhoods of zero which is a fundamental system, and every fundamental system determines a field topology.

1. Definition. A function $p$ from $\omega \times\{0,1\}$ into the set of all finite subsets of $K$ is called a condition, if the following properties hold:

(a) $0 \in p(n, 0)$ and $1 \in p(n, 1)$,

(b) $p(n, 0) \cap p(n, 1)=\varnothing$,

(c) $p(n+1, i) \subset p(n, i)$,

(d) $\varphi_{n}(p(n+1,0)) \subset p(n, 0)$.

Let $P$ be the set of all conditions. $P$ is not empty, since $p^{0}$ defined by $p(n, i)=\{i\}$ for every $n \in \omega$, is an element of $P$. If $p$ and $p^{\prime}$ are two conditions we say that $p^{\prime}$ extends $p$ (written $\left.p \leqq p^{\prime}\right)$, if $p(n, i)$ is a subset of $p^{\prime}(n, i)$ for every $(n, i) \in \omega \times\{0,1\}$. $\leqq$ is a partial ordering of $P$. If $G$ is a chain of conditions, then $V_{n}^{G}$ is defined to be $\bigcup\{p(n, 0) \mid p \in G\}$.

2. THEOREM. Let $G$ be a chain of conditions, then $\left\{V_{n}^{G} \mid n \in \omega\right\}$ is a fundamental system.

The proof is straightforward if we use the fact that

$$
\varphi_{n}(\bigcup\{p(n+1,0) \mid p \in G\})=\bigcup\left\{\varphi_{n}(p(n+1,0)) \mid p \in G\right\} .
$$

For every chain of conditions let $\mathscr{T}_{G}$ denote the field topology which is determined by $\left\{V_{n}^{G} \mid n \in \omega\right\}$.

3. THEOREM. If $\mathscr{T}$ is a field topology with a countable basis, then there is a chain $G$ of conditions such that $\mathscr{T}=\mathscr{T}_{G}$.

Proof. Let $\left\{V_{n} \mid n \in \omega\right\}$ be a fundamental system which determines the topology $\mathscr{T}$ and let $\left(r_{k}^{n}\right)_{k \in \omega}$ be a well ordering of $V_{n}$ for each $n \in \omega$. By recursion we define for every $m$ a condition $p_{m}$ as follows:

$$
\begin{array}{rlrl}
p_{m}(n, i) & =p_{m}(n+1, i) \cup p_{n}\left(p_{m}(n+1, i)\right) \cup\left\{r_{j}^{n} \mid 0 \leqq j \leqq m-n\right\} \\
& =\{i\} \text { otherwise. } & \text { if } n \leqq m \text { and } i=0,
\end{array}
$$

Let $G$ be the set $\left\{p_{m} \mid m \in \omega\right\}$. Then we have for every $n$ that $V_{n}^{G}=V_{n}$ and therefore is $\mathscr{T}_{G}=\mathscr{T}$.

3. Extensions of conditions. Here we prove that for every condition $p$, for each $(n, i) \in \omega \times\{0,1\}$ and for nearly all (this means that for all but finitely many) $r \in K$ there exists a condition $p_{r} \geqq p$ such that $r \in p_{r}(n, i)$. This will enable us to prove in $\S 3$ that there are "many" different chains of conditions. First the easy case.

4. THEOREM. Let $p$ be a condition and $n \in \omega$. Then for nearly all $r \in K$ there is a condition $p_{r}$ such that $p_{r} \geqq p$ and $r \in p_{r}(n, 1)$. 
Proof. Let $r \in K, r \notin p(0,0)$. If we define $p_{r}$ as follows:

$$
\begin{aligned}
p_{r}(n, i) & =p(n, i) \cup\{r\} & & \text { if } i=1, \\
& =p(n, i) & & \text { otherwise, }
\end{aligned}
$$

then $p_{r}$ has the desired properties. Since $p(0,0)$ is finite, this holds for nearly all $r$.

Now the other case. Let $R(K)$ be the set of all rational functions over $K$. If $H$ is a subset of $R(K)$ and if for each $f \in H, r \in K$ is in the domain of $f$, then $H(r)$ shall denote the set of all $f(r)$, with $f \in H$. Let $f \in R(K)$ and $a$ $\in K$ such that $f(0) \neq a$. Then for nearly all $r \in K, f(r) \neq a$. So we obtain:

5. Lemma. Let $H \subset R(K)$ and $M \subset K$ be finite. If $H(0) \cap M=\varnothing$, then for nearly all $r \in K, H(r) \cap M=\varnothing$.

6. Lemma. Let $\varphi \in \phi_{K}$ and let $H$ be a finite subset of $R(K)$ with $1 \notin H(0)$, then there is a finite $H^{\prime} \subset R(K)$ such that, for nearly all $r \in K, \varphi(H(r))=$ $H^{\prime}(r)$.

Proof. If $\varphi=\varphi_{d}$ we define $H^{\prime}$ to be $\{f /(1-g) \mid f, g \in H\}$. Since $1 \notin H(0)$ we have for nearly all $r \in K, 1 \notin H(r)$. Let $r$ be such an element of $K$. Then $\varphi_{d}(H(r))=H(r) /(1-H(r))=H^{\prime}(r)$. Thus, for nearly all $r \in K, \varphi_{d}(H(r))=$ $H^{\prime}(r)$. The proof is similar if $\varphi=\varphi_{c}, \varphi_{b}$ or $\varphi_{a}$.

Now let $p \in P$ and let $H$ be a finite subset of $R(K)$. By induction over $n$ we can prove

7. THEOREM. If $H(0)$ is a subset of $p(n, 0)$, then for nearly all $r \in K$ there is a condition $p_{r} \geqq p$ such that:

(1) $H(r) \subset p_{r}(n, 0)$,

(2) $p_{r}(m, i)=p(m, i)$ if $m>n$ or $i=1$.

(i) The Theorem holds for $n=0$.

Proof. Since $H(0)$ is a subset of $p(n, 0), H(0) \cap p(0,1)=\varnothing$. By Lemma 5 , we have for nearly all $r \in K$ that $p(0,1) \cap H(r)=\varnothing$. Let $r$ be such an element. If $p_{r}$ is defined by

$$
\begin{aligned}
p_{r}(m, i) & =p(m, i) \cup H(r) & & \text { if } m=0 \text { and } \\
& =p(m, i) & & \text { otherwise, }
\end{aligned}
$$

then $p_{r}$ has the desired properties.

(ii) Assume the Theorem holds for $n$, then it holds for $n+1$.

Proof. First we choose a finite subset $H^{\prime \prime \prime}$ of $R(K)$, with $H^{\prime \prime \prime}(0) \subset p(n, 0)$ as follows: Let $H^{\prime}=H \cup\left\{f_{a} \mid a \in p(n+1,0)\right\}$, where $f_{a}$ is the function defined by $f_{a}(r)=a$ for every $r \in K$. Since $1 \notin p(n+1,0)$ and $H^{\prime}(0) \subset$ $p(n+1,0)$, we have by Lemma 6 that there is a finite $H^{\prime \prime} \subset R(K)$ such that 
for nearly all $r \in K, H^{\prime \prime}(r)=\varphi_{n}\left(H^{\prime}(r)\right)$. Let $L$ be the set of these $r$ 's. If we define $H^{\prime \prime \prime}=H^{\prime} \cup H^{\prime \prime}$, then we have that $H^{\prime \prime \prime}(0) \subset p(n, 0)$. By assumption there are for nearly all $r \in K$ conditions $p_{r}^{\prime} \geqq p$ such that:

(1) $H^{\prime \prime \prime}(r) \subset p_{r}^{\prime}(n, 0)$,

(2) $p(m, i)=p_{r}^{\prime}(m, i)$ if $m>n$ or $i=1$.

Let $L^{\prime}$ be the set of these $r$ 's and let $r \in L \cap L^{\prime}$. Then we define $p_{r}$ by

$$
\begin{aligned}
p_{r}(m, i) & =p_{r}^{\prime}(m, i) \cup H(r) & & \text { if } m=n+1 \text { and } i=0, \\
& =p_{r}^{\prime}(m, i) & & \text { otherwise. }
\end{aligned}
$$

$p_{r}$ has the desired properties. Since nearly all $r \in K$ are in $L \cap L^{\prime}$, the theorem holds for $n+1$.

8. Corollary. Let $p$ be a condition and $n \in \omega$. Then for nearly all $r \in K$ there are conditions $p_{r} \geqq p$, such that $r \in p_{r}(n, 0)$.

Proof. Take $H$ to be $\{$ id $\}$, where id is the function which maps every element of $K$ onto itself. By Theorem 7 we get the desired result.

4. Entwined sets of chains of conditions. To prove that there are $2^{2 N_{0}}$ many field topologies on $K$, it suffices to show that there is a set $\mathbb{G}$ of power $2^{N_{0}}$ of chains of conditions such that for any two different nonempty subsets $\mathfrak{F}_{1}$ and $\mathfrak{G}_{2}$ of $\mathfrak{E}$ the join-topologies $\bigvee\left\{\mathscr{T}_{G} \mid G \in \mathfrak{G}_{1}\right\}$ and $\bigvee\left\{\mathscr{T}_{G} \mid G \in \mathfrak{G}_{2}\right\}$ are also different.

9. Definition. Let $(5$ be a set of chains of conditions. $(5$ is called entwined if, for every $n \in \omega$ and for every finite subset $\left\{G_{i} \mid 0 \leqq i \leqq m\right\}$ of $\mathfrak{G}$, there are conditions $p_{i} \in G_{i}, 0 \leqq i \leqq m$, such that

$$
\bigcap\left\{p_{i}(n, 0) \mid 1 \leqq i \leqq m\right\} \cap p_{0}(0,1) \quad \text { is not empty. }
$$

An easy consequence of Definition 9 is that there is a sequence which converges to zero in all of the topologies $\mathscr{T}_{G_{1}}, \cdots, \mathscr{T}_{G_{m}}$ but which is bounded away from zero in $\mathscr{T}_{G_{0}}$.

10. THEOREM. If $(5)$ is entwined, then $\bigvee\left\{\mathscr{T}_{G} \mid G \in \mathfrak{G} \backslash\left\{G_{0}\right\}\right\}$ is not finer than $\mathscr{T}_{G_{0}}$ for every $G_{0} \in \mathfrak{G}$.

Proof. Suppose $\bigvee\left\{\mathscr{T}_{G} \mid G \in \mathscr{G} \backslash\left\{G_{0}\right\}\right\}$ is finer than $\mathscr{T}_{G_{0}}$ for some $G_{0} \in \mathfrak{G}$. Then there are $G_{1}, \cdots, G_{m} \in \mathbb{G}$ and $k_{1}, \ldots, k_{m} \in \omega$ such that

$$
\cap\left\{V_{k i}^{G_{i}} \mid 1 \leqq i \leqq m\right\} \subset V_{0}^{G_{0}} \text {. }
$$

Let $k_{0}=\max \left\{k_{i} \mid 1 \leqq i \leqq m\right\}$. Since $\left(5\right.$ is entwined, there are conditions $p_{i} \in G_{i}$, $0 \leqq i \leqq m$, such that $M=\bigcap\left\{p_{i}\left(k_{0}, 0\right) \mid 1 \leqq i \leqq m\right\} \cap p_{0}(0,1)$ is not empty. If $r \in \bar{M}$, then $r \in V_{k_{0}}^{G_{i}} \subset V_{k_{i}}^{G_{i}}$ and $r \in V_{0}^{G_{0}}$. This is a contradiction. 
11. Corollary. Let $\mathfrak{G}_{5}$ be entwined and $\mathfrak{5}_{1}, \mathfrak{5}_{2}$ nonempty different subsets of $\mathfrak{G}_{\text {. Then }} \bigvee\left\{\mathscr{T}_{G} \mid G \in \mathfrak{G}_{1}\right\}$ and $\bigvee\left\{\mathscr{T}_{G} \mid G \in \mathfrak{G}_{2}\right\}$ are different.

Proof. Let $\mathfrak{F}_{1}, \mathfrak{G}_{2} \subset \mathfrak{F}$ such that $\mathfrak{F}_{1} \neq \mathfrak{G}_{2}$. We may suppose that there is a $G_{0} \in \mathfrak{G}_{1}$ with $G_{0} \notin \mathfrak{F}_{2}$. Because $\mathfrak{G}$ is entwined $\bigvee\left\{\mathscr{T}_{G} \mid G \in \mathfrak{G} \backslash\left\{G_{0}\right\}\right\}$ is not finer than $\mathscr{T}_{G_{0}} . \bigvee\left\{\mathscr{T}_{G}|G \in \mathscr{G}|\left\{G_{0}\right\}\right\}$ is finer than $\bigvee\left\{\mathscr{T}_{G} \mid G \in \mathfrak{G}_{2}\right\}$ and $\bigvee\left\{\mathscr{T}_{G} \mid G \in \mathfrak{F}_{1}\right\}$ is finer than $\mathscr{T}_{G_{0}}$. Thus, $\bigvee\left\{\mathscr{T}_{G} \mid G \in \mathscr{G}_{2}\right\}$ is not finer than $\bigvee\left\{\mathscr{T}_{G} \mid G \in \mathfrak{G}_{1}\right\}$.

Now we want to show that there is an entwined 65 of power $2^{N_{0}}$. We identify each natural number with the set of its predecessors. ${ }^{n} 2$ denotes the set of functions from $n$ into 2 and ${ }^{\omega} 2$ the set of those from $\omega$ into 2 . If $f$ is such a function, then $f \mid n$ is the restriction of $f$ to $n$. By induction over $n$, we shall choose, for each $f \in^{n+1} 2$, a condition $p^{f}$ such that:

(1) $p^{f \mid n} \leqq p^{f}$.

(2) $p^{f}(0,1) \cap \bigcap\left\{p^{g}(n, 0) \mid g \in{ }^{n+1} 2\right.$ and $\left.g \neq f\right\}$ is not empty.

(3) There is an $r \in \bigcap\left\{p^{g}(n, 0) \mid g \in{ }^{n+1} 2\right\}$ and an $m \in \omega, m \neq 0$, such that $r^{m} \in \bigcap\left\{p^{g}(0,1) \mid g \in{ }^{n+1} 2\right\}$.

Let $p^{\phi}=p^{0}$. Assume that, for $f \in{ }^{n} 2, p^{f}$ is chosen. Let $\left(f_{k}\right)_{k \in m_{n}}$ be a well ordering of ${ }^{n+1} 2$. For every $f \in{ }^{n+1} 2$ we choose, by induction over $k \in$ $m_{n}+1$ conditions, $p_{k}^{f}$ as follows: By Corollary 8 there are conditions $p_{*}^{f}$ such that $p_{*}^{f} \geqq p^{\left.f\right|^{n}}$ and $M=\bigcap\left\{p_{*}^{f}(n, 0) \mid f \in{ }^{n+1} 2\right\} \backslash\{0\} \neq \varnothing$. Let $r \in M$ be given. If there is an $m \in \omega$ such that $r^{m}=1$, then take $p_{0}^{f}$ to be $p_{*}^{f}$. If there is no $m \in \omega$ such that $r^{m}=1$, then $\left\{r^{m} \mid m \in \omega\right\}$ is infinite. Hence, by Theorem 4 there are conditions $p_{0}^{f} \geqq p_{*}^{f}$ and an $m \in \omega$ such that $r^{m} \in$ $\bigcap\left\{p_{0}^{f}(0,1) \mid f \in \in^{n+1} 2\right\}$.

Suppose $p_{k}^{f}$ is already chosen. Then by Theorem 4 and Corollary 8 there are conditions $p_{k+1}^{f}$ such that $p_{k+1}^{f} \geqq p_{k}^{f}$ and $p_{k+1}^{f_{k}}(0,1) \cap \bigcap\left\{p_{k+1}^{f}(n, 0)\right.$ $f \in{ }^{n+1} 2$ and $\left.f \neq f_{k}\right\}$ is nonempty. Let $p^{f}=p_{m_{n}}^{f}$. Then the conditions $p^{f}$, $f \in{ }^{n+1} 2$, have the desired properties. Now for $g \in{ }^{\omega} 2$, define $G_{g}$ to be the chain $\left\{p^{g \hat{\mid} \mid n} \mid n \in \omega\right\}$, and define $\left(5\right.$ to be $\left\{G_{g} \mid g \in{ }^{\omega} 2\right\}$.

12. THEOREM. (5 is an entwined set of power $2^{N_{0}}$ of chains of conditions.

Proof. It is sufficient to show that 65 is entwined. Let $g_{0} \in{ }^{\omega} 2$ and let $g_{i} \in{ }^{\omega} 2 \backslash\left\{g_{0}\right\}, 1 \leqq i \leqq m$. Then for each $n$ there is a $k>n$ such that $g_{0} \mid k \notin$ $\left\{g_{i}|k| 1 \leqq i \leqq m\right\}$. By 2 , we know that $p^{g_{0} \mid k}(0,1) \cap \bigcap\left\{p^{g_{i} \mid k}(k-1,0) \mid\right.$ $1 \leqq i \leqq m\}$ is not empty. Since $k-1 \geqq n$ we have that $p^{\left.g_{i}\right|_{k}}(k-1,0)$ is a subset of $p^{g_{i} \hat{\mid}}(n, 0)$. This implies that $p^{g_{0} \mid k}(0,1) \cap \bigcap\left\{p^{\left.g_{i}\right\}_{k}}(n, 0) \mid 1 \leqq i \leqq m\right\}$ is not empty. Thus, $(5$ is entwined.

Now we shall prove that we have constructed $\mathfrak{G}$ in such a way that for each $\mathfrak{G}_{1} \subset \mathfrak{G}$ the topology $\bigvee\left\{\mathscr{T}_{G} \mid G \in \mathfrak{F}_{1}\right\}$ is not the join of locally bounded ring topologies. By [1] it is sufficient to show that there is a neighborhood $V$ of zero such that for every neighborhood $U \subset V$ there is an $n \in \omega$ with $U^{n} \notin V$. 
13. THEOREM. For every $\mathfrak{G}^{\prime} \subset \mathfrak{b}, \bigvee\left\{\mathscr{T}_{G} \mid G \in \mathfrak{G}^{\prime}\right\}$ fails to be the join of locally bounded ring topologies.

Proof. Let $G_{0} \in \mathfrak{G}^{\prime}$ be given and let $U$ be a neighborhood of zero, $U \subset V_{0}^{G_{0}}$. Then there are finitely many $G_{i} \in \mathfrak{G}^{\prime}, 1 \leqq i \leqq m$, and a $k \in \omega$ such that $\bigcap\left\{V_{k}^{G_{i}} 1 \leqq i \leqq m\right\} \subset U$. By the definition of $(5)$ there are functions $f_{i} \in{ }^{k+1} 2,0 \leqq i \leqq m$, such that $p^{f_{i}}(k, 0) \subset V_{k}^{G_{i}}$. From 3 it follows that there is an $r \in \bigcap\left\{p^{f_{i}}(k, 0) \mid 1 \leqq i \leqq m\right\}$ and a $z \in \omega, z \neq 0$, such that $r^{z} \in p^{f_{0}}(0,1)$. Hence, $U^{z} 屯 V_{0}^{G_{0}}$ and therefore $\bigvee\left\{\mathscr{T}_{G} \mid G \in \mathfrak{b}^{\prime}\right\}$ is not the join of locally bounded ring topologies.

\section{REFERENCES}

1. J. Heine, Existence of locally unbounded topological fields, J. London Math. Soc. (2) 5 (1972), 481-487.

2. J. O. Kiltinen, Inductive ring topologies, Trans. Amer. Math. Soc. 134 (1968), 149-169. MR 37 \#4054.

3. H.-J. Kowalsky, Beiträge zur topologischen Algebra, Math. Nachr. 11 (1954), 143-185. MR 15, 774.

InStitut Für Mathematik, TeChNische Universität, HanNover, Welfengarten 1, HANNOVER, West GeRMANY 